\title{
Evidence synthesis for sustainability
}

\author{
The volume of work contributing substantial understanding and new evidence about sustainability challenges is \\ growing. Making the most of it is imperative for interventions to be really effective.
}

S ustainability scholarship, practice and policy encompass a huge variety of needs, skills and experiences. Sustainability challenges are varied and complex as we all know. They are also pressing and increasingly so. This in turn means that growing knowledge and evidence are being produced and disseminated through various channels including academic journals and grey literature documents among others. Keeping abreast of such a growing amount of research can be daunting. In addition, some of these valuable documents often including the outcomes of policy evaluations are shelved, once complete, and remain unread. In one way or another, available knowledge risks not having the impact it should - producing syntheses of such knowledge and evidence does enhance taking stock and moving forward. Ultimately, it does make a real difference in how research money is invested and interventions and policies designed. Robust syntheses can help understand multifaceted problems such as, for example, improving sanitation conditions and achieving food security in developing regions. It helps in identifying critical knowledge gaps, and, when it comes to interventions and policies it allows learning lessons about what worked and what didn't. Ultimately, society stands to gain from all this. So, there is no doubt, robust evidence synthesis should be promoted.

There is a long tradition of evidence synthesis in the medical sciences which is where robust tools and methods have been developed and are now well established. The output of evidence syntheses can take various forms, including systematic reviews, meta-analyses, scoping reviews, rapid evidence assessments, systematic maps and a lot more. Among the many types of evidence synthesis, systematic reviews have been the most widely used so far and are well known for rigour, breadth and impact. Systematic reviews as defined by the Cochrane Collaboration address clearly defined research questions and follow systematic and explicit methods to select the relevant literature, and collect and analyse data from the studies selected. In order to ensure a robust and replicable approach, these synthesis articles follow strict reporting guidelines, such as those set out by the PRISMA statement. In short, these syntheses represent original research efforts developed

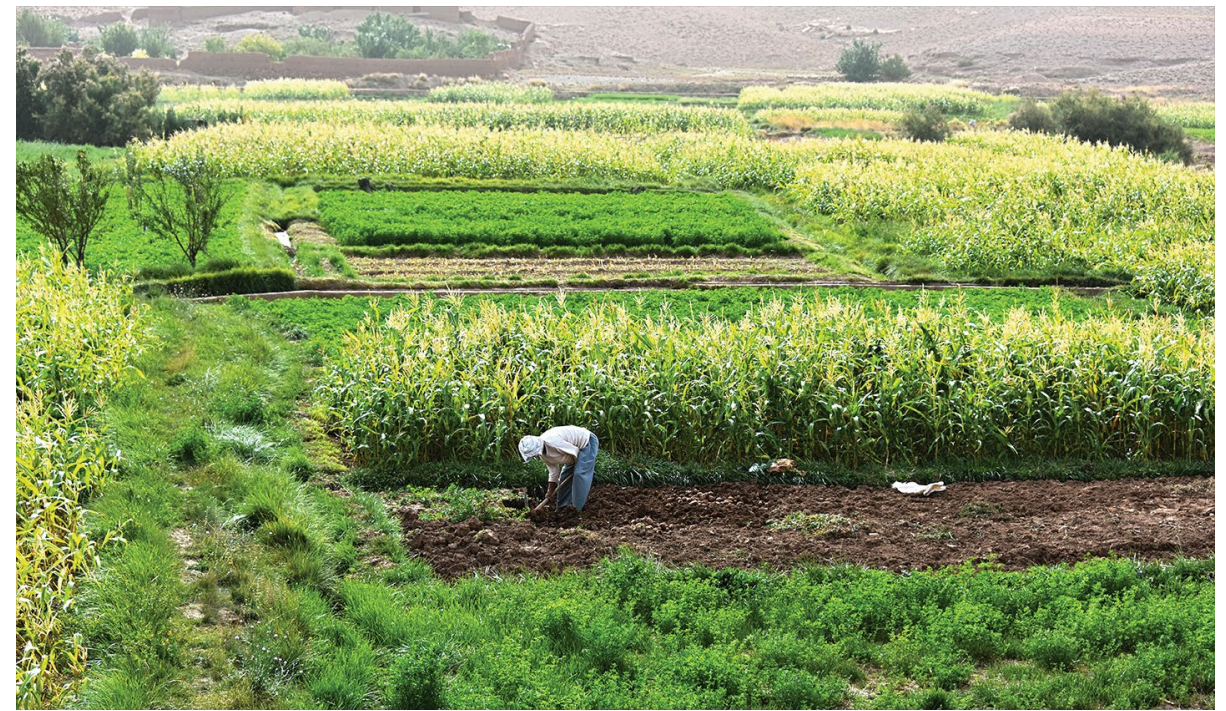

Credit: Zoonar GmbH / Alamy Stock Photo

following structured protocols preregistered and publicly available before the actual synthesis articles are developed.

Though quite popular approaches to evidence synthesis, systematic reviews carry some costs. In particular, they are extremely resource demanding. In addition, they might not work in domains where data are not consistently and continuously collected or when interventions vary substantially in design, as is the case for example in the context of sustainable development. Other types of evidence synthesis can be more suitable. One emerging article is the scoping review - useful to scope a body of published work in order to identify gaps or to clarify concepts, or even confirm the importance of potential questions, among other things. In this issue, Nature Sustainability publishes four such examples contributing knowledge synthesis to various dimensions of Sustainable Development Goal 2, zero hunger. An article by Liverpool-Tasie et al. sheds light on whether interactions not formalized through contracts between small farmers and both small- and large-scale value chain actors have impacts on small farmers' livelihoods. Another article by Piñeiro and colleagues analyses whether or not incentive-based programmes lead to the adoption of sustainable practices and what the effects are on environmental, economic and productivity outcomes. Stathers and co-authors focus on analysing the kind of interventions designed to reduce postharvest losses of food crops in sub-Saharan Africa and South Asia. Finally, Ricciardi and colleagues map the existing literature about on-farm interventions that improve the incomes or yields of small-scale farmers in water scarce regions. These scoping reviews, included in a collection (https:// www.nature.com/collections/end-hunger) jointly with Nature Food and Nature Plants, are the outcome of an innovative and broad effort, including the work of more than 70 researchers from 25 countries, to evaluate agricultural interventions that have potential to sustainably improve the living conditions of among the poorer farmers around the world.

Increasing the use of evidence synthesis for, and adapting methods and types of syntheses to, sustainability-relevant research questions, including about biodiversity conservation programmes, climate change policies and natural resource management strategies, will further knowledge about the complexity of human-nature interfaces and improve the design and effectiveness of interventions. A lot is already happening, but more work is needed. We hope that going forward more resources and scholarly interest will support the development of worthwhile evidence synthesis efforts that contribute to change practices in view of achieving a better future for all.

Published online: 13 October 2020

https://doi.org/10.1038/s41893-020-00629-8 